\title{
Pacific
}

Journal of

Mathematics

\section{THE YAMABE PROBLEM ON NONCOMPACT CR MANIFOLDS}

\section{Pak Tung Ho and Seongtag Kim}




\title{
THE YAMABE PROBLEM ON NONCOMPACT CR MANIFOLDS
}

\author{
PAK TUng Ho And SeOngtag Kim
}

\begin{abstract}
Let $(M, \theta)$ be a noncompact complete strictly pseudoconvex $C R$ manifold of real dimension $2 n+1 \geq 3$ with positive Webster scalar curvature. We show that there exists a conformal contact form $\tilde{\theta}=u^{2 / n} \theta$ with positive constant Webster scalar curvature if the CR-Yamabe invariant $Y(M, \theta)$ of $(M, \theta)$ is positive and strictly less than the CR-Yamabe invariant at infinity $\overline{Y(M, \theta)}$.
\end{abstract}

\section{Introduction}

Suppose that $(M, g)$ is a compact Riemannian manifold of dimension $n \geq 3$. As a generalization of the uniformization theorem, the Yamabe problem is to find a metric conformal to $g$ such that its scalar curvature is constant. This was solved by Trudinger [1968], Aubin [1976] and Schoen [1984]. The uniqueness of the solution of the Yamabe problem was studied in [Kazdan and Warner 1975; Lou 1998]. See the survey article [Lee and Parker 1987] for more about the Yamabe problem. See also [Brendle 2005; 2007; Chow 1992; Schwetlick and Struwe 2003; Ye 1994] for results related to the Yamabe flow, which is the geometric flow introduced to study the Yamabe problem.

The Yamabe problem was also studied on complete noncompact Riemannian manifolds. In this case, there is a simple counterexample such that the Yamabe problem does not have a solution (see [Jin 1988]). See also [Aviles and McOwen 1988; Bland and Kalka 1989; Große and Nardmann 2014; Kim 1997; 2000; Zhang 2003] and references therein for results related to the Yamabe problem on noncompact Riemannian manifolds. In particular, we mention the following result which is related to our main theorem. If $(M, g)$ is a noncompact Riemannian manifold with positive scalar curvature $R_{g}$, we define

$$
Y(M, g)=\inf _{u \in C_{0}^{\infty}(M)} \frac{\int_{M}\left|\nabla_{g} u\right|^{2}+\frac{n-2}{4(n-1)} R_{g} u^{2} d V_{g}}{\left(\int_{M} u^{\frac{2 n}{n-2}} d V_{\theta}\right)^{\frac{n-2}{n}}}
$$

MSC2010: primary 32V20, 53C21; secondary 32V05, 58J05.

Keywords: CR manifolds, CR Yamabe problem, Webster scalar curvature, conformal changes, noncompact complete manifolds. 
and

$$
\overline{Y(M, g)}=\lim _{r \rightarrow \infty} \inf _{u \in C_{0}^{\infty}\left(M-B_{r}\right)} \frac{\int_{M-B_{r}}\left|\nabla_{g} u\right|^{2}+\frac{n-2}{4(n-1)} R_{g} u^{2} d V_{g}}{\left(\int_{M-B_{r}} u^{\frac{2 n}{n-2}} d V_{g}\right)^{\frac{n-2}{n}}},
$$

where $r$ is the distance induced by the Riemannian metric $g$ from $x$ to a fixed point $x_{0}$ in $M$, and $B_{r}$ is the ball of radius $r$ centered at $x_{0}$. The second author [Kim 1996] proved the following:

Theorem 1.1. Suppose $(M, g)$ is a noncompact Riemannian manifold with positive scalar curvature with $0<Y(M, g)<\overline{Y(M, g)}$. Then there exists a Riemannian metric conformal to $g$ which has positive constant scalar curvature.

The Yamabe problem can also be formulated in the context of CR manifolds. Suppose that $(M, \theta)$ is a compact strictly pseudoconvex CR manifold of real dimension $2 n+1$ with a given contact form $\theta$. The CR Yamabe problem is to find a contact form conformal to $\theta$ such that its Webster scalar curvature is constant. This was introduced by Jerison and Lee [1987], and was solved by them for the case when $n \geq 2$ and $M$ is not locally CR equivalent to the sphere $S^{2 n+1}$ in [Jerison and Lee 1987; 1988; 1989]. The remaining cases, namely when $n=1$ or when $M$ is locally CR equivalent to the sphere, were studied respectively in [Gamara and Yacoub 2001] and in [Gamara 2001]. See also the recent work of Cheng, Chiu and Yang [Cheng et al. 2014] and Cheng, Malchiodi and Yang [Cheng et al. 2013] for the study of these two cases. The uniqueness of the solution of the CR Yamabe problem was studied in [Ho 2013; Jerison and Lee 1987]. On the other hand, the CR Yamabe flow, the geometric flow introduced to study the CR Yamabe problem, was studied in [Chang and Cheng 2002; Chang et al. 2010; Ho 2012; 2015].

In this paper, we study the CR Yamabe problem on noncompact manifolds. We suppose that $(M, \theta)$ is a noncompact strictly pseudoconvex CR manifold of real dimension $2 n+1$ such that its Webster scalar curvature $R_{\theta}$ is positive. We would like to find another contact form conformal to $\theta$ such that its Webster scalar curvature is constant. This is equivalent to finding a positive solution to the equation

$$
-\Delta_{\theta} u+\frac{n}{2 n+2} R_{\theta} u=q u^{1+\frac{2}{n}},
$$

where $q$ is a positive constant. We define

$$
Y(M, \theta)=\inf _{u \in C_{0}^{\infty}(M)} \frac{\int_{M}\left|\nabla_{\theta} u\right|^{2}+\frac{n}{2 n+2} R_{\theta} u^{2} d V_{\theta}}{\left(\int_{M} u^{2+\frac{2}{n}} d V_{\theta}\right)^{\frac{n}{n+1}}}
$$


and

$$
\overline{Y(M, \theta)}=\lim _{r \rightarrow \infty} \inf _{u \in C_{0}^{\infty}\left(M-B_{r}\right)} \frac{\int_{M-B_{r}}\left|\nabla_{\theta} u\right|^{2}+\frac{n}{2 n+2} R_{\theta} u^{2} d V_{\theta}}{\left(\int_{M-B_{r}} u^{2+\frac{2}{n}} d V_{\theta}\right)^{\frac{n}{n+1}}},
$$

where $\nabla_{\theta}$ is the subgradient with respect to $\theta, d V_{\theta}=\theta \wedge(d \theta)^{n}$ is the volume form of $\theta, r$ is the Carnot-Carathéodory distance from $x$ to a fixed point $x_{0} \in M$ with respect to the contact form $\theta$, and $B_{r}$ is the ball of radius $r$ centered at $x_{0}$. We refer readers to the book [Dragomir and Tomassini 2006] or the paper [Jerison and Lee 1987] for more about the definitions and concepts related to CR manifolds.

Note that $\overline{Y(M, \theta)}$ is well defined. Indeed, if we let

$$
f(r)=\inf _{u \in C_{0}^{\infty}\left(M-B_{r}\right)} \frac{\int_{M-B_{r}}\left|\nabla_{\theta} u\right|^{2}+\frac{n}{2 n+2} R_{\theta} u^{2} d V_{\theta}}{\left(\int_{M-B_{r}} u^{2+\frac{2}{n}} d V_{\theta}\right)^{\frac{n}{n+1}}},
$$

then it follows from the definition that $f(r)$ is nondecreasing as a function of $r$. Since $f(r)$ is bounded above by $Y\left(S^{2 n+1}, \theta_{S^{2 n+1}}\right), \lim _{r \rightarrow \infty} f(r)$ exists.

The following is our main theorem, which is the CR version of Theorem 1.1.

Theorem 1.2. Let $(M, \theta)$ be a noncompact strictly pseudoconvex $C R$ manifold of real dimension $2 n+1 \geq 3$ with positive Webster scalar curvature. Assume that

$$
0<Y(M, \theta)<\overline{Y(M, \theta)} .
$$

Then there exists a positive solution $u$ of (1-1). That is, the contact form $u^{2 / n_{\theta}}$ conformal to $\theta$ has positive constant Webster scalar curvature.

\section{Proof}

Since we have assumed that

$$
0<Y(M, \theta)<\overline{Y(M, \theta)}\left(\leq Y\left(S^{2 n+1}, \theta_{S^{2 n+1}}\right)\right)
$$

there exists a sequence of smooth compact domains $K_{i}$ such that $Y\left(K_{i}, \theta\right)<$ $\overline{Y(M, \theta)}$ with $K_{i} \subset K_{i+1}$ satisfying $\cup K_{i}=M$. Using the work on the CR Yamabe problem in the compact case (see [Jerison and Lee 1987]) for $2 n+1 \geq 3$, we have a positive smooth function $u_{i}$ on each $K_{i}$ with

$$
-\Delta_{\theta} u_{i}+\frac{n}{2 n+2} R_{\theta} u_{i}=q_{i} u_{i}^{1+\frac{2}{n}} \text { on } K_{i}
$$


$u_{i}=0$ on $\partial K_{i}$ and

$$
\int_{K_{i}} u_{i}^{2+\frac{2}{n}} d V_{\theta}=1
$$

where

$$
q_{i}=Y\left(K_{i}, \theta\right) \rightarrow Y(M, \theta) \quad \text { as } i \rightarrow \infty
$$

We extend the domain of $u_{i}$ by defining $u_{i}=0$ outside $K_{i}$, and we still denote its extension by $u_{i}$. Then the extension of $u_{i}$ is in $S_{1}^{2}(M, \theta)$, the completion of $C_{0}^{\infty}(M)$ with the norm

$$
\|u\|_{S_{1}^{2}(M, \theta)}^{2}=\int_{M}\left|\nabla_{\theta} u\right|^{2}+\frac{n}{2 n+2} R_{\theta} u^{2} d V_{\theta} .
$$

For sufficiently large $i$, let $\widetilde{K}$ and $K^{\prime}$ be fixed smooth compact subsets of $M$ with $\widetilde{K} \subset K^{\prime} \subset K_{i}$. We shall show that $\int_{\widetilde{K}} u_{i}^{(1+b)(2+2 / n)} d V_{\theta}$ is uniformly bounded for some positive $b$. The constant $c(\epsilon)$ in the Sobolev embedding for $u_{i}$ on a noncompact complete Riemannian manifold depends on the domain and does not have to be uniformly bounded (see (2-7)); therefore the Sobolev embedding is not directly applicable in (6) of [Kim 1996]. However, the Sobolev embedding holds for $u_{i} \varphi$ on a fixed domain $K^{\prime}$, where $\varphi$ is a cutoff function supported in $K^{\prime}$. The uniform bound of $u_{i}$ in $L_{(1+b)(2+2 / n)}(\tilde{K})$ can be obtained on each compact subset $\widetilde{K}$, by applying the same method of [Kim 1996] to $u_{i} \varphi$. The detailed proof for the CR case is provided in the following steps.

Take

$$
\Omega=\left\{x \in K^{\prime} \mid u_{i}(x) \geq 1\right\}
$$

with

$$
Y\left(K^{\prime}, \theta\right)<Y\left(S^{2 n+1}, \theta_{S^{2 n+1}}\right) .
$$

Then

$$
|\Omega|=\int_{\Omega} d V_{\theta}<1
$$

by (2-2). Now let $u_{i}=1+w_{i}$. Then

$$
\Omega=\left\{x \in K^{\prime} \mid w_{i}(x) \geq 0\right\}
$$

by definition, for sufficiently large $i$, and (2-1) is equivalent to

$$
-\Delta_{\theta} w_{i}+\frac{n}{2 n+2} R_{\theta}\left(1+w_{i}\right)=q_{i}\left(1+w_{i}\right)^{1+\frac{2}{n}} \quad \text { on } K_{i} .
$$


Take a smooth cutoff function $\varphi \in C_{0}^{\infty}\left(K^{\prime}\right)$ with $\varphi \equiv 1$ on $\tilde{K}$ and $|\varphi| \leq 1$ on $K^{\prime}$. Multiplying (2-5) by $\varphi^{2+2 b} w_{i}^{1+2 b}$, where $b>0$, and integrating it over $\Omega$, we get (2-6)

$$
\begin{aligned}
q_{i} \int_{\Omega} \varphi^{2+2 b} w_{i}^{1+2 b}\left(1+w_{i}\right)^{1+\frac{2}{n}} d V_{\theta} \\
=-\int_{\Omega} \varphi^{2+2 b} w_{i}^{1+2 b} \Delta_{\theta} w_{i} d V_{\theta}+\frac{n}{2 n+2} \int_{\Omega} R_{\theta}\left(1+w_{i}\right) \varphi^{2+2 b} w_{i}^{1+2 b} d V_{\theta} \\
=\int_{\Omega} \frac{1+2 b}{(1+b)^{2}} \varphi^{2+2 b}\left|\nabla_{\theta}\left(w_{i}^{1+b}\right)\right|^{2}+\frac{2}{1+b} \varphi^{1+b} w_{i}^{1+b} \nabla_{\theta} w_{i}^{1+b} \cdot \nabla_{\theta} \varphi^{1+b} d V_{\theta} \\
\quad+\frac{n}{2 n+2} \int_{\Omega} R_{\theta}\left(1+w_{i}\right) w_{i}^{1+2 b} \varphi^{2+2 b} d V_{\theta} \\
\geq \int_{\Omega} \frac{1+2 b-\epsilon_{1}}{(1+b)^{2}} \varphi^{2+2 b}\left|\nabla_{\theta}\left(w_{i}^{1+b}\right)\right|^{2}-\frac{1}{\epsilon_{1}}\left|\nabla_{\theta} \varphi^{1+b}\right|^{2} w_{i}^{2+2 b} d V_{\theta} \\
\quad+\frac{n}{2 n+2} \int_{\Omega} R_{\theta}\left(1+w_{i}\right) w_{i}^{1+2 b} \varphi^{2+2 b} d V_{\theta},
\end{aligned}
$$

where we used integration by parts, Hölder's inequality and (2-4).

We are going to estimate the terms on the right-hand side of (2-6). Applying (A-1) in the Appendix for $\varphi w_{i} \in C_{0}^{\infty}(\Omega)$, where $\Omega \subset K_{i} \subset M$, we obtain that for any given $\epsilon>0$, there exists $C(\epsilon)$, which depends on the given domain $\Omega$, such that

$$
\begin{aligned}
& \left(\int_{\Omega}\left(\varphi^{1+b} w_{i}^{1+b}\right)^{2+\frac{2}{n}} d V_{\theta}\right)^{\frac{n}{n+1}} \\
& \leq \frac{1+\epsilon}{Y\left(S^{2 n+1}, \theta_{S^{2 n+1}}\right)} \int_{\Omega}\left|\nabla_{\theta}\left(\varphi^{1+b} w_{i}^{1+b}\right)\right|^{2} d V_{\theta}+C(\epsilon) \int_{\Omega}\left(\varphi^{1+b} w_{i}^{1+b}\right)^{2} d V_{\theta} \\
& \leq \frac{1+\epsilon}{Y\left(S^{2 n+1}, \theta_{S^{2 n+1}}\right)} \int_{\Omega}\left(1+\epsilon_{2}\right) \varphi^{2+2 b}\left|\nabla_{\theta} w_{i}^{1+b}\right|^{2} \\
& \quad+\frac{1}{\epsilon_{2}} w_{i}^{2+2 b}\left|\nabla_{\theta} \varphi^{1+b}\right|^{2} d V_{\theta}+C(\epsilon) \int_{\Omega}\left(\varphi^{1+b} w_{i}^{1+b}\right)^{2} d V_{\theta} \\
& \quad \times \frac{1+\epsilon}{Y\left(S^{2 n+1}, \theta_{S^{2 n+1}}\right)}\left(\int_{\Omega} \frac{(1+b)^{2}\left(1+\epsilon_{2}\right)}{1+2 b-\epsilon_{1}}-\frac{n}{2 n+2} R_{\theta}\left(1+w_{i}\right) w_{i}^{1+2 b} \varphi^{2+2 b}\right) \\
& \left.+C(\epsilon) \int_{\Omega}^{1+2 b}\left(\varphi^{1+b} w_{i}^{1+b}\right)^{2} d V_{\theta}, \quad+\frac{1}{\epsilon_{2}} w_{i}^{2+2 b}\left|\nabla_{\theta} \varphi^{1+b}\right|^{2} d V_{\theta}\right)
\end{aligned}
$$


where we have used (2-6) in the last inequality. Now let $\Omega_{1}=\left\{x \in \Omega \mid w_{i} \geq 2\right\}$ and $\Omega_{2}=\Omega-\Omega_{1}$. Let $a=1+2 / n$ and $x=1 / w_{i}$. Note that if $w_{i} \in \Omega_{1}$, i.e., $w_{i} \geq 2$, then $|x| \leq \frac{1}{2}$ and

$$
\begin{aligned}
\left(1+w_{i}\right)^{a}-w_{i}^{a} & =w_{i}^{a}\left(1+\frac{1}{w_{i}}\right)^{a}-w_{i}^{a} \\
& =w_{i}^{a}(1+x)^{a}-w_{i}^{a} \\
& =w_{i}^{a}\left(1+a x+\frac{1}{2} a(a-1) x^{2}+\cdots-1\right) \\
& \leq c_{1} w_{i}^{2 / n}
\end{aligned}
$$

for some constant $c_{1}$. Using (2-8), the integral in (2-7) can be estimated as follows:

$$
\begin{gathered}
\left(\int_{\Omega}\left(\varphi^{1+b} w_{i}^{1+b}\right)^{2+\frac{2}{n}} d V_{\theta}\right)^{\frac{n}{n+1}} \\
\leq \frac{1+\epsilon}{Y\left(S^{2 n+1}, \theta_{S^{2 n+1}}\right)}\left(\frac{(1+b)^{2}\left(1+\epsilon_{2}\right)}{1+2 b-\epsilon_{1}}\right. \\
\times\left(\int_{\Omega_{1}} q_{i} \varphi^{2+2 b} w_{i}^{2+2 b+\frac{2}{n}}+C \varphi^{2+2 b} w_{i}^{1+2 b+\frac{2}{n}} d V_{\theta}\right. \\
+\int_{\Omega_{2}} q_{i} \varphi^{2+2 b} w_{i}^{1+2 b}\left(1+w_{i}\right)^{1+\frac{2}{n}} d V_{\theta} \\
\quad+\int_{\Omega} \frac{1}{\epsilon_{1}}\left|\nabla_{\theta} \varphi^{1+b}\right|^{2} w_{i}^{2+2 b} \\
\left.-\frac{n}{2 n+2} R_{\theta}\left(1+w_{i}\right) w_{i}^{1+2 b} \varphi^{2+2 b} d V_{\theta}\right) \\
\left.+C(\epsilon) \int_{\Omega}\left(\varphi^{1+b} w_{i}^{1+b}\right)^{2} d V_{\theta} \cdot \int_{\Omega} \frac{1}{\epsilon_{2}} w_{i}^{2+2 b}\left|\nabla_{\theta} \varphi^{1+b}\right|^{2} d V_{\theta}\right)
\end{gathered}
$$

By Hölder's inequality, we have

$$
\begin{aligned}
\int_{\Omega} \varphi^{2+2 b} & w_{i}^{2+2 b+\frac{2}{n}} d V_{\theta} \\
& \leq\left(\int_{\Omega}\left(\varphi^{1+b} w_{i}^{1+b}\right)^{2+\frac{2}{n}} d V_{\theta}\right)^{\frac{n}{n+1}}\left(\int_{\Omega} w_{i}^{\frac{2}{n}(n+1)} d V_{\theta}\right)^{\frac{1}{n+1}} \\
& \leq\left(\int_{\Omega}\left(\varphi^{1+b} w_{i}^{1+b}\right)^{2+\frac{2}{n}} d V_{\theta}\right)^{\frac{n}{n+1}}
\end{aligned}
$$


where the last inequality follows from

$$
\int_{\Omega} w_{i}^{2+\frac{2}{n}} d V_{\theta} \leq \int_{\Omega} u_{i}^{2+\frac{2}{n}} d V_{\theta} \leq 1
$$

by (2-2) and the definition of $w_{i}$ and $\Omega$. Since $\Omega_{1} \subset \Omega$, we can combine (2-9) and (2-10) to get

$(2-11)$

$$
\begin{aligned}
& \left(\int_{\Omega}\left(\varphi^{1+b} w_{i}^{1+b}\right)^{2+\frac{2}{n}} d V_{\theta}\right)^{\frac{n}{n+1}} \\
& \leq \frac{1+\epsilon}{Y\left(S^{2 n+1}, \theta_{\left.S^{2 n+1}\right)}\right.} \\
& \quad \times\left(\frac{(1+b)^{2}\left(1+\epsilon_{2}\right)}{1+2 b-\epsilon_{1}}\right. \\
& \quad \times\left(q_{i}\left(\int_{\Omega}\left(\varphi^{1+b} w_{i}^{1+b}\right)^{2+\frac{2}{n}} d V_{\theta}\right)^{\frac{n}{n+1}}+C \int_{\Omega_{1}} \varphi^{2+2 b} w_{i}^{1+2 b+\frac{2}{n}} d V_{\theta}\right. \\
& \quad+\int_{\Omega_{2}} q_{i} \varphi^{2+2 b} w_{i}^{1+2 b}\left(1+w_{i}\right)^{1+\frac{2}{n}} d V_{\theta}+\int_{\Omega} \frac{1}{\epsilon_{1}}\left|\nabla_{\theta} \varphi^{1+b}\right|^{2} w_{i}^{2+2 b} \\
& \left.\quad-\frac{n}{2 n+2} R_{\theta}\left(1+w_{i}\right) w_{i}^{1+2 b} \varphi^{2+2 b} d V_{\theta}\right) \\
& +C(\epsilon) \int_{\Omega}\left(\varphi^{1+b} w_{i}^{1+b}\right)^{2} d V_{\theta} .
\end{aligned}
$$

Since $q_{i}<Y\left(K^{\prime}, \theta\right)<\overline{Y(M, \theta)} \leq Y\left(S^{2 n+1}, \theta_{S^{2 n+1}}\right)$, we can take $\epsilon, \epsilon_{1}, \epsilon_{2}$ and $0<b<1 / n$ sufficiently small such that

$$
\frac{1+\epsilon}{Y\left(S^{2 n+1}, \theta_{S^{2 n+1}}\right)} \frac{(1+b)^{2}\left(1+\epsilon_{2}\right)}{1+2 b-\epsilon_{1}} q_{i} \leq c_{0}<1 .
$$

Combining this with (2-11), we obtain

$$
\begin{array}{r}
\left(1-c_{0}\right)\left(\int_{\Omega}\left(\varphi w_{i}\right)^{(1+b)(2+2 / n)} d V_{\theta}\right)^{\frac{n}{n+1}}+C \int_{\Omega} R_{\theta}\left(1+w_{i}\right) w_{i}^{1+2 b} \varphi^{2+2 b} d V_{\theta} \\
\leq C \int_{\Omega_{1}} w_{i}^{1+\frac{2}{n}+2 b} \varphi^{2+2 b} d V_{\theta}+C \int_{\Omega_{2}} w_{i}^{1+2 b}\left(1+w_{i}\right)^{1+\frac{2}{n}} \varphi^{2+2 b} d V_{\theta} \\
+C \int_{\Omega}\left(w_{i}^{1+b}\right)^{2} \varphi^{2+2 b} d V_{\theta}+C \int_{\Omega}\left|\nabla_{\theta} \varphi^{1+b}\right|^{2} w_{i}^{2+2 b} d V_{\theta}
\end{array}
$$


Here $C$ is a constant independent of $i$. We are going to estimate the terms on the right-hand side of (2-12). Since $\Omega_{2}=\Omega-\Omega_{1}$, we have

$$
\left|\Omega_{2}\right|<1 \quad \text { and } \quad 0 \leq w_{i} \leq 2 \quad \text { on } \Omega_{2} .
$$

This implies that

$$
\int_{\Omega_{2}} w_{i}^{1+2 b}\left(1+w_{i}\right)^{1+\frac{2}{n}} \varphi^{2+2 b} d V_{\theta} \leq C
$$

for some constant $C$ independent of $i$. Also, since $b<1 / n$, we have

$$
t_{1}:=\frac{1+b}{1+\frac{1}{n}}<1
$$

Then it follows from Hölder's inequality that

$$
\int_{\Omega}\left(w_{i}^{1+b}\right)^{2} \varphi^{2+2 b} d V_{\theta} \leq\left(\int_{\Omega} w_{i}^{2+\frac{2}{n}} d V_{\theta}\right)^{t_{1}}|\Omega|^{1-t_{1}} \leq\left(\int_{\Omega} u_{i}^{2+\frac{2}{n}} d V_{\theta}\right)^{t_{1}} \leq 1,
$$

where we have used (2-2), (2-3) and (2-4). On the other hand, since $w_{i} \geq 2$ in $\Omega_{1}$ and $b<1 / n$, we have

$$
\int_{\Omega_{1}} w_{i}^{1+\frac{2}{n}+2 b} \varphi^{2+2 b} d V_{\theta} \leq \int_{\Omega_{1}} w_{i}^{2+\frac{2}{n}} d V_{\theta} \leq \int_{\Omega} u_{i}^{2+\frac{2}{n}} d V_{\theta} \leq 1,
$$

where we have used (2-2). Since $\varphi$ is a smooth fixed cutoff function, the last term of (2-12) is also bounded. Combining all these, we can conclude that the right-hand side of (2-12) is uniformly bounded. Thus, the left-hand side of (2-12) is uniformly bounded; i.e.,

$\left(1-c_{0}\right)\left(\int_{\Omega}\left(\varphi w_{i}\right)^{(1+b)(2+2 / n)} d V_{\theta}\right)^{\frac{n}{n+1}}+C \int_{\Omega} R_{\theta}\left(1+w_{i}\right) w_{i}^{1+2 b} \varphi^{2+2 b} d V_{\theta} \leq C$.

In particular, this implies that

$$
\left(\int_{\Omega}\left(\varphi w_{i}\right)^{(1+b)(2+2 / n)} d V_{\theta}\right)^{\frac{n}{n+1}} \leq C_{0}
$$

and

$$
\int_{\widetilde{K}} w_{i}^{(1+b)(2+2 / n)} d V_{\theta} \leq C_{0}^{\prime}
$$

for some constants $C_{0}$ and $C_{0}^{\prime}$ independent of $i$. Therefore, $u_{i}$ is uniformly bounded in $L_{(1+b)(2+2 / n)}(\widetilde{K})$ for each compact subset $\widetilde{K}$ of $M$ and some positive $b$.

We can now show that $w_{i}$ is $C^{2, \alpha}$ bounded on each compact subset of $M$ in the following way: Consider sufficiently large compact subsets $K \subset K_{0} \subset K_{1} \subset K_{2}$ 
with smooth boundary satisfying $Y(K, \theta)<Y\left(S^{2 n+1}, \theta_{S^{2 n+1}}\right)$. It follows from (2-14) that

$$
\int_{K_{2}} w_{i}^{2+\frac{2}{n}+2 \bar{b}} d V_{\theta} \leq C_{0},
$$

where $\bar{b}=b(1+1 / n)$ and $C_{0}$ is a constant independent of $i$. Also, we have

$$
\left|\Delta_{\theta} w_{i}\right|=\left|\frac{n}{2 n+2} R_{\theta}\left(1+w_{i}\right)-q_{i}\left(1+w_{i}\right)^{1+\frac{2}{n}}\right| \leq C\left(1+w_{i}\right)^{1+\frac{2}{n}} \quad \text { on } K_{2},
$$

where $C$ is a constant that depends only on $K_{2}$ and $\max _{K_{2}} R_{\theta}$. Hence, $\Delta_{\theta} w_{i} \in$ $L^{q}\left(K_{2}\right)$, where $q=(2 n+2+2 n \bar{b}) /(n+2)$. By the regularity theory (see [Jerison and Lee 1987, Proposition 5.7(c)]), we have $w_{i} \in S_{2}^{q}\left(K_{1}\right)$. From the FollandStein embedding theorem (see [Jerison and Lee 1987, Proposition 5.5]), we have $w_{i} \in L^{s}\left(K_{1}\right)$, where

$$
s=\left(2+\frac{2}{n}+2 \bar{b}\right) \frac{n+1}{n+1-2 \bar{b}}>2+\frac{2}{n}+2 \bar{b} .
$$

Continuing this procedure, we get $w_{i} \in S_{2}^{t}\left(K_{0}\right)$ for all $t>1$. Again by the FollandStein embedding theorem (see [Jerison and Lee 1987, Proposition 5.7(a-b)]), we have $w_{i}^{2+2 / n} \in C^{\alpha}\left(K_{0}\right)$ for some $\alpha>0$. By the regularity theory again (see [Jerison and Lee 1987, Proposition 5.9(b)]), we can conclude that $w_{i} \in C^{2, \alpha}(K)$, as required.

By the definition of $\Omega$ and since $u_{i}=1+w_{i}$, we have a uniform $C^{2, \alpha}$ bound for $u_{i}$ on each compact subset of $M$. Therefore, we can find a subsequence, which we still denote by $\left\{u_{i}\right\}$, that converges to some $u$ uniformly on each compact subset by the Arzelà-Ascoli theorem.

To sum up, we have proved the following:

Lemma 2.1. If $Y(M, \theta)<\overline{Y(M, \theta)}$, then there exists a subsequence $\left\{u_{i}\right\}$ which converges to a solution $u$ of (1-1) uniformly on each compact subset of $M$.

We remark that we do not know whether $u$ is strictly positive. Note that if $u=0$ at some point of $M$, then by applying Proposition 2.2 (stated below) to (1-1), we can conclude that $u$ is identically equal to zero.

Proposition 2.2. Suppose that $u$ is a nonnegative function on $M$ satisfying

$$
-\Delta_{\theta} u+P(x) u \geq 0,
$$

where $P(x)$ is a smooth function on $M$. Then for any compact set $K$ in $M$, there exists a constant $C$ such that

$$
\int_{K} u^{2+\frac{2}{n}} d V_{\theta} \leq C\left(\min _{K} u\right)\left(\max _{K} u\right)^{\frac{n+2}{n}} .
$$


We skip the proof of Proposition 2.2, because it is essentially the same as the proof of Proposition A.1 in [Ho 2012].

We are going to show that it is impossible for $u$ to be identically equal to zero. First, we have the following:

Lemma 2.3. As $i \rightarrow \infty$,

$$
\int_{M}\left|u_{i}\right|^{2+\frac{2}{n}} d V_{\theta}-\int_{M}\left|u-u_{i}\right|^{2+\frac{2}{n}} d V_{\theta} \rightarrow \int_{M}|u|^{2+\frac{2}{n}} d V_{\theta}
$$

Proof. Note that

$$
\begin{aligned}
& \int_{M}\left|u_{i}\right|^{2+\frac{2}{n}} d V_{\theta}-\int_{M}\left|u-u_{i}\right|^{2+\frac{2}{n}} d V_{\theta} \\
&=-\int_{M} \int_{0}^{1} \frac{\partial}{\partial t}\left|u_{i}-t u\right|^{2+\frac{2}{n}} d t d V_{\theta} \\
&=\left(2+\frac{2}{n}\right) \int_{M} \int_{0}^{1} u\left(u_{i}-t u\right)\left|u_{i}-t u\right|^{\frac{2}{n}} d t d V_{\theta} \\
& \rightarrow\left(2+\frac{2}{n}\right) \int_{M} \int_{0}^{1} u(u-t u)|u-t u|^{\frac{2}{n}} d t d V_{\theta} \\
&=\int_{M}|u|^{2+\frac{2}{n}} d V_{\theta}
\end{aligned}
$$

as $i \rightarrow \infty$.

For abbreviation, we let

$$
v_{i}=u_{i}-u \quad \text { and } \quad E(v)=\int_{M}\left(\left|\nabla_{\theta} v\right|^{2}+\frac{n}{2 n+2} R_{\theta} v^{2}\right) d V_{\theta} .
$$

Lemma 2.4. As $i \rightarrow \infty$,

$$
E\left(u_{i}\right)-E\left(v_{i}\right) \rightarrow E(u) .
$$

Proof. We compute

$$
\begin{aligned}
E\left(u_{i}\right)-E\left(v_{i}\right) & =E\left(u+v_{i}\right)-E\left(v_{i}\right) \\
& =E(u)+2 \int_{M}\left(-\Delta_{\theta} u+\frac{n}{2 n+2} R_{\theta} u\right) v_{i} d V_{\theta} \\
& \rightarrow E(u)
\end{aligned}
$$

as $i \rightarrow \infty$, since $v_{i}$ tends to 0 weakly in $S_{1}^{2}(M)$. This proves the assertion.

Lemma 2.5. For any fixed $B_{r}$, we have

$$
E\left(v_{i}\right) \geq Y\left(M-B_{r}, \theta\right)\left(\int_{M-B_{r}}\left|v_{i}\right|^{2+\frac{2}{n}} d V_{\theta}\right)^{\frac{n}{n+1}}+o(1) \quad \text { as } i \rightarrow \infty .
$$


Proof. Note that

$$
\begin{aligned}
E\left(v_{i}\right) & =\int_{M}\left(\left|\nabla_{\theta} v_{i}\right|^{2}+\frac{n}{2 n+2} R_{\theta} v_{i}^{2}\right) d V_{\theta} \\
& =\int_{M-B_{r}}\left(\left|\nabla_{\theta} v_{i}\right|^{2}+\frac{n}{2 n+2} R_{\theta} v_{i}^{2}\right) d V_{\theta}+\int_{B_{r}}\left(\left|\nabla_{\theta} v_{i}\right|^{2}+\frac{n}{2 n+2} R_{\theta} v_{i}^{2}\right) d V_{\theta} \\
& \geq \int_{M-B_{r}}\left(\left|\nabla_{\theta} v_{i}\right|^{2}+\frac{n}{2 n+2} R_{\theta} v_{i}^{2}\right) d V_{\theta}+o(1) \\
& \geq Y\left(M-B_{r}, \theta\right)\left(\int_{M-B_{r}}\left|v_{i}\right|^{2+\frac{2}{n}} d V_{\theta}\right)^{\frac{n}{n+1}}+o(1),
\end{aligned}
$$

where the first inequality follows from the fact that $v_{i} \rightarrow 0$ uniformly on $B_{r}$ by Lemma 2.1. This proves the assertion.

Note that $u_{i} \rightarrow u$ weakly in $S_{1}^{2}(M, \theta)$. Assume that

$$
\int_{M}|u|^{2+2 / n} d V_{\theta}=\lambda
$$

Note that if $\lambda>0$, then

$$
E(u)=\lambda^{\frac{n}{n+1}} E\left(\lambda^{-\frac{n}{2 n+2}} u\right) \geq \lambda^{\frac{n}{n+1}} Y(M, \theta) .
$$

Furthermore, if $\lambda<1$, then

(2-16) $\quad E\left(v_{i}\right)=(1-\lambda)^{\frac{n}{n+1}} E\left((1-\lambda)^{-\frac{n}{2 n+2}} v_{i}\right) \geq(1-\lambda)^{\frac{n}{n+1}} \overline{Y(M, \theta)}+O(1)$

by the definition of $\overline{Y(M, \theta)}$.

We have the following three cases:

Case 1. If $0<\lambda<1$, then

$$
\begin{aligned}
Y(M, \theta) & =E\left(u_{i}\right)+o(1) \\
& =E(u)+E\left(v_{i}\right)+o(1) \\
& \geq \lambda^{\frac{n}{n+1}} Y(M, \theta)+(1-\lambda)^{\frac{n}{n+1}} \overline{Y(M, \theta)}+o(1) \\
& \geq\left(\lambda^{\frac{n}{n+1}}+(1-\lambda)^{\frac{n}{n+1}}\right) Y(M, \theta)+o(1),
\end{aligned}
$$

where the second equality follows from Lemma 2.4, the first inequality follows from (2-15) and (2-16), and the last inequality follows from the assumption that $Y(M, \theta)<\overline{Y(M, \theta)}$. But this is a contradiction, since

$$
\lambda^{t}+(1-\lambda)^{t}>(1-\lambda+\lambda)^{t}=1 \quad \text { for } 0<\lambda<1 \text { and } 0<t<1 .
$$


Case 2. If $\lambda=0$, then

$$
\begin{aligned}
Y(M, \theta) & =E\left(u_{i}\right)+o(1) \\
& =E(u)+E\left(v_{i}\right)+o(1) \\
& \geq E\left(v_{i}\right)+o(1) \\
& \geq \overline{Y(M, \theta)}+o(1),
\end{aligned}
$$

where the second equality follows from Lemma 2.4 , and the last inequality follows from (2-16) with $\lambda=0$. But this contradicts the assumption that $Y(M, \theta)<$ $\overline{Y(M, \theta)}$.

Case 3. Therefore, we must have $\lambda=1$; i.e.,

$$
\int_{M}|u|^{2+\frac{2}{n}} d V_{\theta}=1
$$

This implies that $u$ is not identically equal to zero. As pointed out in the remark after Lemma 2.1, $u$ is strictly positive. Therefore, we have a positive solution $u$ in $S_{1}^{2}(M, \theta)$ for $(1-1)$.

Now it follows from Theorem 5.15 in [Jerison and Lee 1987] that $u$ is smooth. This proves Theorem 1.2.

\section{Appendix}

We prove the following inequality related to the Folland-Stein embedding:

Theorem A.1. Suppose $K$ is a smooth compact subset in $M$. For any $\epsilon>0$, there exists a constant $C(\epsilon, K)$ such that

$$
\begin{aligned}
& \text { (A-1) } Y\left(S^{2 n+1}, \theta_{S^{2 n+1}}\right)\left(\int_{K}|\varphi|^{2+\frac{2}{n}} d V_{\theta}\right)^{\frac{n}{n+1}} \\
& \leq(1+\epsilon) \int_{K}\left|\nabla_{\theta} \varphi\right|^{2} d V_{\theta}+C(\epsilon, K) \int_{K}|\varphi|^{2} d V_{\theta}
\end{aligned}
$$

for all $\varphi \in S_{1}^{2}(M, \theta)$ with its compact support lying in $K$.

We remark that Theorem A.1 is probably well known. But we cannot find it in the literature. Therefore we provide the proof here. In particular, the Riemannian version of Theorem A.1 can be found in Theorem 2.21 of [Aubin 1998].

Proof of Theorem A.1. Given any $\delta>0$, for any point $p \in M$, there exists a neighborhood $U_{p}$ of $p$ and a diffeomorphism $f_{p}$ from $U_{p}$ to a neighborhood of the origin of $\mathbb{H}^{n}$ such that (see [Jerison and Lee 1987, Theorem 4.3])

$$
\begin{aligned}
\left(f_{p}\right)_{*}\left(d V_{\theta}\right) & =(1+O(\delta)) d V_{\theta_{H^{n}}}, \\
\left(f_{p}\right)_{*}\left(\left|\nabla_{\theta} \varphi\right|^{2}\right) & =(1+O(\delta))\left|\nabla_{\theta_{H^{n}}}(\varphi \circ f)\right|^{2}
\end{aligned}
$$


for any function $\varphi$ in $M$. It follows from [Jerison and Lee 1988, Corollary C] that

$$
\left(\int_{\mathbb{H}^{n}}|\varphi|^{2+\frac{2}{n}} d V_{\theta_{\uplus^{n}}}\right)^{\frac{n}{n+1}} \leq K(n, 2) \int_{\mathbb{H}^{n}}\left|\nabla_{\theta_{\uplus^{n}}} \varphi\right|^{2} d V_{\theta_{\uplus^{n}}}
$$

for any smooth function $\varphi$ which has compact support in $\mathbb{H}^{n}$, where

$$
\begin{aligned}
K(n, 2) & =\frac{1}{2 \pi n(n+1)} \\
& =\frac{1}{Y\left(S^{2 n+1}, \theta_{S^{2 n+1}}\right)} .
\end{aligned}
$$

This implies that (A-3) is also true for $\varphi \in S_{1}^{2}\left(\mathbb{\boxplus}^{n}, \theta_{\mathbb{W}^{n}}\right)$ which is compactly supported. Combining (A-2) and (A-3), we get

$$
\begin{aligned}
\left(\int_{U_{p}}|\varphi|^{2+\frac{2}{n}} d V_{\theta}\right)^{\frac{n}{n+1}} & =\left(\int_{f_{p}\left(U_{p}\right)}\left|\varphi \circ f_{p}\right|^{2+\frac{2}{n}}\left(f_{p}\right)_{*}\left(d V_{\theta}\right)\right)^{\frac{n}{n+1}} \\
& \leq(1+O(\delta))\left(\int_{f_{p}\left(U_{p}\right)}\left|\varphi \circ f_{p}\right|^{2+\frac{2}{n}} d V_{\theta_{H^{n}}}\right)^{\frac{n}{n+1}} \\
& \leq(1+O(\delta)) K(n, 2) \int_{f_{p}\left(U_{p}\right)}\left|\nabla_{\theta_{\uplus} n}\left(\varphi \circ f_{p}\right)\right|^{2} d V_{\theta_{\sharp} n} \\
& \leq(1+O(\delta)) K(n, 2) \int_{U_{p}}\left|\nabla_{\theta} \varphi\right|^{2} d V_{\theta}
\end{aligned}
$$

for any function $\varphi$ which has compact support in $U_{p}$.

Since $K$ is compact, there exists a finite subcovering $\left\{U_{p_{i}}\right\}_{i=1}^{k}$; i.e.,

$$
K=\bigcup_{i=1}^{k} U_{p_{i}}
$$

Suppose $\left\{h_{i}\right\}_{i=1}^{k}$ is a partition of unity subordinate to $\left\{U_{p_{i}}\right\}_{i=1}^{k}$; i.e., the support of $h_{i}$ lies in $U_{p_{i}}$,

$$
\sum_{i=1}^{k} h_{i}=1 \quad \text { and } \quad\left|\nabla_{\theta}\left(h_{i}^{1 / 2}\right)\right| \leq H .
$$

For abbreviation, we write

$$
\|\varphi\|_{p}=\left(\int_{M}|\varphi|^{p} d V_{\theta}\right)^{\frac{1}{p}} .
$$


Therefore, for any function $\varphi$ compactly supported in $K$, we have

$$
\begin{aligned}
& \text { (A-6) } \sum_{i=1}^{k}\left\|\varphi^{2} h_{i}\right\|_{\frac{n+1}{n}} \\
&=\sum_{i=1}^{k}\left\|\varphi h_{i}^{1 / 2}\right\|_{2+\frac{2}{n}}^{2} \\
& \leq(1+O(\delta)) K(n, 2) \sum_{i=1}^{k}\left\|\nabla_{\theta}\left(\varphi h_{i}^{1 / 2}\right)\right\|_{2}^{2} \\
& \leq \\
& \leq(1+O(\delta)) K(n, 2) \sum_{i=1}^{k} \int\left(\left|\nabla_{\theta} \varphi\right| h_{i}^{1 / 2}+\varphi\left|\nabla_{\theta}\left(h_{i}^{1 / 2}\right)\right|\right)^{2} d V_{\theta} \\
& \quad \times \int \sum_{i=1}^{k}\left(\left|\nabla_{\theta} \varphi\right|^{2} h_{i}+2\left|\nabla_{\theta} \varphi\right| h_{i}^{1 / 2}\left|\varphi \| \nabla_{\theta}\left(h_{i}^{1 / 2}\right)\right|+|\varphi|^{2}\left|\nabla_{\theta}\left(h_{i}^{1 / 2}\right)\right|^{2}\right) d V_{\theta} \\
& \leq(1+O(\delta)) K(n, 2)\left(\left\|\nabla_{\theta} \varphi\right\|_{2}^{2}+2 k H\left\|\nabla_{\theta} \varphi\right\|_{2}\|\varphi\|_{2}+k H\|\varphi\|_{2}^{2}\right),
\end{aligned}
$$

where the first inequality follows from (A-4), the last inequality follows from (A-5) and

$$
\left(\sum_{i=1}^{k} h_{i}^{1 / 2}\right)^{2} \leq k \sum_{i=1}^{k} h_{i}=k
$$

by Hölder's inequality.

For any $\epsilon>0$, we can choose $\delta$ small enough such that

$$
(1+O(\delta)) K(n, 2) \leq K(n, 2)+\frac{\epsilon}{2} .
$$

Since the last expression of (A-6) is independent of $i$, we establish the inequality

$$
\begin{aligned}
\|\varphi\|_{2+\frac{2}{n}}^{2}=\left\|\varphi^{2}\right\|_{\frac{n+1}{n}} & =\left\|\varphi^{2} \sum_{i=1}^{k} h_{i}\right\|_{\frac{n+1}{n}} \\
& \leq \sum_{i=1}^{k}\left\|\varphi^{2} h_{i}\right\|_{\frac{n+1}{n}} \\
& \leq(1+O(\delta)) K(n, 2)\left(\left\|\nabla_{\theta} \varphi\right\|_{2}^{2}+2 k H\left\|\nabla_{\theta} \varphi\right\|_{2}\|\varphi\|_{2}+k H\|\varphi\|_{2}^{2}\right) \\
& \leq\left(K(n, 2)+\frac{\epsilon}{2}\right)\left(\left\|\nabla_{\theta} \varphi\right\|_{2}^{2}+2 k H\left\|\nabla_{\theta} \varphi\right\|_{2}\|\varphi\|_{2}+k H\|\varphi\|_{2}^{2}\right) \\
& \leq\left(K(n, 2)+\frac{\epsilon}{2}\right)\left((1+\epsilon)\left\|\nabla_{\theta} \varphi\right\|_{2}^{2}+C(\epsilon, k, H)\|\varphi\|_{2}^{2}\right),
\end{aligned}
$$


where we have used (A-7) and Young's inequality. Here $C(\epsilon, k, H)$ is a constant depending only on $\epsilon, k$ and $H$. This proves the assertion.

\section{Acknowledgements}

The authors are grateful to the referee for valuable comments which improved the manuscript. Ho was supported by a National Research Foundation of Korea (NRF) grant funded by the Korean government (MEST) (No. 201531021.01) and Kim was supported by the Basic Science Research Program through the National Research Foundation of Korea (NRF) funded by the Ministry of Education, Science and Technology (NRF-2011-0025674).

\section{References}

[Aubin 1976] T. Aubin, "Équations différentielles non linéaires et problème de Yamabe concernant la courbure scalaire", J. Math. Pures Appl. (9) 55:3 (1976), 269-296. MR 0431287 Zbl 0336.53033

[Aubin 1998] T. Aubin, Some nonlinear problems in Riemannian geometry, Springer, Berlin, 1998. MR 1636569 Zbl 0896.53003

[Aviles and McOwen 1988] P. Aviles and R. C. McOwen, "Conformal deformation to constant negative scalar curvature on noncompact Riemannian manifolds", J. Differential Geom. 27:2 (1988), 225-239. MR 925121 Zbl 0648.53021

[Bland and Kalka 1989] J. Bland and M. Kalka, "Negative scalar curvature metrics on noncompact manifolds", Trans. Amer. Math. Soc. 316:2 (1989), 433-446. MR 987159 Zbl 0694.53041

[Brendle 2005] S. Brendle, "Convergence of the Yamabe flow for arbitrary initial energy", J. Differential Geom. 69:2 (2005), 217-278. MR 2168505 Zbl 1085.53028

[Brendle 2007] S. Brendle, "Convergence of the Yamabe flow in dimension 6 and higher", Invent. Math. 170:3 (2007), 541-576. MR 2357502 Zbl 1130.53044

[Chang and Cheng 2002] S.-C. Chang and J.-H. Cheng, "The Harnack estimate for the Yamabe flow on CR manifolds of dimension 3", Ann. Global Anal. Geom. 21:2 (2002), 111-121. MR 1894940 Zbl 1007.53034

[Chang et al. 2010] S.-C. Chang, H.-L. Chiu, and C.-T. Wu, "The Li-Yau-Hamilton inequality for Yamabe flow on a closed CR 3-manifold", Trans. Amer. Math. Soc. 362:4 (2010), 1681-1698. MR 2574873 Zbl 1192.32020

[Cheng et al. 2013] J.-H. Cheng, A. Malchiodi, and P. Yang, "A positive mass theorem in three dimensional Cauchy-Riemann geometry”, preprint, 2013. arXiv 1312.7764

[Cheng et al. 2014] J.-H. Cheng, H.-L. Chiu, and P. Yang, "Uniformization of spherical CR manifolds", Adv. Math. 255 (2014), 182-216. MR 3167481 Zbl 1288.32051

[Chow 1992] B. Chow, "The Yamabe flow on locally conformally flat manifolds with positive Ricci curvature”, Comm. Pure Appl. Math. 45:8 (1992), 1003-1014. MR 1168117 Zbl 0785.53027

[Dragomir and Tomassini 2006] S. Dragomir and G. Tomassini, Differential geometry and analysis on CR manifolds, Progress in Mathematics 246, Birkhäuser, Boston, 2006. MR 2214654 Zbl 1099.32008

[Gamara 2001] N. Gamara, "The CR Yamabe conjecture - the case $n=1$ ", J. Eur. Math. Soc. (JEMS) 3:2 (2001), 105-137. MR 1831872 Zbl 0988.53013 
[Gamara and Yacoub 2001] N. Gamara and R. Yacoub, "CR Yamabe conjecture - the conformally flat case”, Pacific J. Math. 201:1 (2001), 121-175. MR 1867895 Zbl 1054.32020

[Große and Nardmann 2014] N. Große and M. Nardmann, "The Yamabe constant on noncompact manifolds", J. Geom. Anal. 24:2 (2014), 1092-1125. MR 3192307 Zbl 1315.53028

[Ho 2012] P. T. Ho, "The long-time existence and convergence of the CR Yamabe flow", Commun. Contemp. Math. 14:2 (2012), 1250014, 50. MR 2901057 Zbl 1246.53087

[Ho 2013] P. T. Ho, "Results related to prescribing pseudo-Hermitian scalar curvature", Internat. J. Math. 24:3 (2013), 1350020, 29. MR 3048007 Zbl 1267.32034

[Ho 2015] P. T. Ho, “The Webster scalar curvature flow on CR sphere, I”, Adv. Math. 268 (2015), 758-835. MR 3276608 Zbl 1301.32028

[Jerison and Lee 1987] D. Jerison and J. M. Lee, "The Yamabe problem on CR manifolds", $J$. Differential Geom. 25:2 (1987), 167-197. MR 880182 Zbl 0661.32026

[Jerison and Lee 1988] D. Jerison and J. M. Lee, "Extremals for the Sobolev inequality on the Heisenberg group and the CR Yamabe problem", J. Amer. Math. Soc. 1:1 (1988), 1-13. MR 924699 Zbl 0634.32016

[Jerison and Lee 1989] D. Jerison and J. M. Lee, "Intrinsic CR normal coordinates and the CR Yamabe problem”, J. Differential Geom. 29:2 (1989), 303-343. MR 982177 Zbl 0671.32016

[Jin 1988] Z. R. Jin, "A counterexample to the Yamabe problem for complete noncompact manifolds", pp. 93-101 in Partial differential equations (Tianjin, 1986), edited by S. S. Chern, Lecture Notes in Math. 1306, Springer, 1988. MR 1032773 Zbl 0648.53022

[Kazdan and Warner 1975] J. L. Kazdan and F. W. Warner, "Existence and conformal deformation of metrics with prescribed Gaussian and scalar curvatures", Ann. of Math. (2) 101 (1975), 317-331. MR 0375153 Zbl 0297.53020

[Kim 1996] S. Kim, "Scalar curvature on noncompact complete Riemannian manifolds", Nonlinear Anal. 26:12 (1996), 1985-1993. MR 1386128 Zbl 0858.53029

[Kim 1997] S. Kim, "The Yamabe problem and applications on noncompact complete Riemannian manifolds", Geom. Dedicata 64:3 (1997), 373-381. MR 1440570 Zbl 0878.53037

[Kim 2000] S. Kim, "An obstruction to the conformal compactification of Riemannian manifolds", Proc. Amer. Math. Soc. 128:6 (2000), 1833-1838. MR 1646195 Zbl 0956.53032

[Lee and Parker 1987] J. M. Lee and T. H. Parker, "The Yamabe problem”, Bull. Amer. Math. Soc. (N.S.) 17:1 (1987), 37-91. MR 888880 Zbl 0633.53062

[Lou 1998] Y. Lou, "Uniqueness and non-uniqueness of metrics with prescribed scalar curvature on compact manifolds", Indiana Univ. Math. J. 47:3 (1998), 1065-1081. MR 1665745 Zbl 0936.53028

[Schoen 1984] R. Schoen, "Conformal deformation of a Riemannian metric to constant scalar curvature”, J. Differential Geom. 20:2 (1984), 479-495. MR 788292 Zbl 0576.53028

[Schwetlick and Struwe 2003] H. Schwetlick and M. Struwe, "Convergence of the Yamabe flow for "large” energies", J. Reine Angew. Math. 562 (2003), 59-100. MR 2011332 Zbl 1079.53100

[Trudinger 1968] N. S. Trudinger, "Remarks concerning the conformal deformation of Riemannian structures on compact manifolds", Ann. Scuola Norm. Sup. Pisa (3) 22 (1968), 265-274. MR 0240748 Zbl 0159.23801

[Ye 1994] R. Ye, "Global existence and convergence of Yamabe flow", J. Differential Geom. 39:1 (1994), 35-50. MR 1258912 Zbl 0846.53027

[Zhang 2003] Q. S. Zhang, "Finite energy solutions to the Yamabe equation", Geom. Dedicata 101 (2003), 153-165. MR 2017900 Zbl 1058.58015 
Received February 15, 2016. Revised April 18, 2016.

PAK TUNG Ho

DEPARTMENT OF MATHEMATICS

SOGANG UNIVERSITY

SEOUL 121-742

SOUTH KOREA

ptho@sogang.ac.kr

paktungho@yahoo.com.hk

SeOngtag Kim

DEPARTMENT OF MATHEMATICS EDUCATION

INHA UNIVERSITY

INCHEON 402-751

SOUTH KOREA

stkim@inha.ac.kr 


\title{
PACIFIC JOURNAL OF MATHEMATICS
}

Founded in 1951 by E. F. Beckenbach (1906-1982) and F. Wolf (1904-1989)

$$
\text { msp.org/pjm }
$$

\section{EDITORS}

\author{
Don Blasius (Managing Editor) \\ Department of Mathematics \\ University of California \\ Los Angeles, CA 90095-1555 \\ blasius@math.ucla.edu
}

\author{
Paul Balmer \\ Department of Mathematics \\ University of California \\ Los Angeles, CA 90095-1555 \\ balmer@math.ucla.edu \\ Robert Finn \\ Department of Mathematics \\ Stanford University \\ Stanford, CA 94305-2125 \\ finn@math.stanford.edu \\ Sorin Popa \\ Department of Mathematics \\ University of California \\ Los Angeles, CA 90095-1555 \\ popa@math.ucla.edu
}

\author{
Vyjayanthi Chari \\ Department of Mathematics \\ University of California \\ Riverside, CA 92521-0135 \\ chari@math.ucr.edu \\ Kefeng Liu \\ Department of Mathematics \\ University of California \\ Los Angeles, CA 90095-1555 \\ liu@math.ucla.edu \\ Igor Pak \\ Department of Mathematics \\ University of California \\ Los Angeles, CA 90095-1555 \\ pak.pjm@gmail.com \\ Paul Yang \\ Department of Mathematics \\ Princeton University \\ Princeton NJ 08544-1000 \\ yang@math.princeton.edu
}

\section{PRODUCTION}

Silvio Levy, Scientific Editor, production@msp.org

\section{SUPPORTING INSTITUTIONS}

ACADEMIA SINICA, TAIPEI

CALIFORNIA INST. OF TECHNOLOGY

STANFORD UNIVERSITY

UNIV. OF BRITISH COLUMBIA

UNIV. OF CALIFORNIA, BERKELEY

UNIV. OF CALIFORNIA, DAVIS

UNIV. OF CALIFORNIA, LOS ANGELES

UNIV. OF CALIFORNIA, RIVERSIDE

UNIV. OF CALIFORNIA, SAN DIEGO

UNIV. OF CALIF., SANTA BARBARA
KEIO UNIVERSITY

MATH. SCIENCES RESEARCH INSTITUTE

NEW MEXICO STATE UNIV.

OREGON STATE UNIV.
Daryl Cooper

Department of Mathematics

University of California

Santa Barbara, CA 93106-3080 cooper@math.ucsb.edu

Jiang-Hua Lu

Department of Mathematics

The University of Hong Kong

Pokfulam Rd., Hong Kong

jhlu@maths.hku.hk

$$
\text { Jie Qing }
$$

Department of Mathematics

University of California

Santa Cruz, CA 95064

qing@ cats.ucsc.edu

\author{
UNIV. OF CALIF., SANTA CRUZ \\ UNIV. OF MONTANA \\ UNIV. OF OREGON \\ UNIV. OF SOUTHERN CALIFORNIA \\ UNIV. OF UTAH \\ UNIV. OF WASHINGTON \\ WASHINGTON STATE UNIVERSITY
}

These supporting institutions contribute to the cost of publication of this Journal, but they are not owners or publishers and have no responsibility for its contents or policies.

See inside back cover or msp.org/pjm for submission instructions.

The subscription price for 2016 is US $\$ 440 /$ year for the electronic version, and \$600/year for print and electronic.

Subscriptions, requests for back issues and changes of subscriber address should be sent to Pacific Journal of Mathematics, P.O. Box 4163, Berkeley, CA 94704-0163, U.S.A. The Pacific Journal of Mathematics is indexed by Mathematical Reviews, Zentralblatt MATH, PASCAL CNRS Index, Referativnyi Zhurnal, Current Mathematical Publications and Web of Knowledge (Science Citation Index).

The Pacific Journal of Mathematics (ISSN 0030-8730) at the University of California, c/o Department of Mathematics, 798 Evans Hall \#3840, Berkeley, CA 94720-3840, is published twelve times a year. Periodical rate postage paid at Berkeley, CA 94704, and additional mailing offices. POSTMASTER: send address changes to Pacific Journal of Mathematics, P.O. Box 4163, Berkeley, CA 94704-0163.

PJM peer review and production are managed by EditFLOW ${ }^{\circledR}$ from Mathematical Sciences Publishers.

PUBLISHED BY

\section{I. mathematical sciences publishers}

nonprofit scientific publishing

http://msp.org/

(C) 2016 Mathematical Sciences Publishers 


\section{PACIFIC JOURNAL OF MATHEMATICS}

Volume $285 \quad$ No. $2 \quad$ December 2016

The $\mathrm{SU}(N)$ Casson-Lin invariants for links

HANS U. BODEN and ERIC HARPER

The SU(2) Casson-Lin invariant of the Hopf link

HANS U. BODEN and CHRISTOPHER M. HERALD

Commensurations and metric properties of Houghton's groups

José BuRILlo, SEAn Cleary, ARMANDo MaRTino and ClaAs

E. RÖVER

Conformal holonomy equals ambient holonomy

ANDREAS ČAP, A. RoD GOVER, C. RoBIN GRAHAM and

MATTHIAS HAMMERL

Nonorientable Lagrangian cobordisms between Legendrian knots

ORSOLA CAPOVILLA-SEARLE and LISA TRAYNOR

A strong multiplicity one theorem for $\mathrm{SL}_{2}$

JINGSONG CHAI and QING ZHANG

The Yamabe problem on noncompact CR manifolds

PAK TUNG Ho and SeONGTAG KIM

Isometry types of frame bundles

WOUTER VAN LIMBEEK

Bundles of spectra and algebraic K-theory

JOHN A. LIND

Hidden symmetries and commensurability of 2-bridge link complements

CHRISTIAN MiLlichaP and WILLIAM WORDEN

On seaweed subalgebras and meander graphs in type $C$

DMITRI I. PANYUSHEV and OKSANA S. YAKIMOVA

The genus filtration in the smooth concordance group 